\title{
Stochastic Gravitational Wave Background from Coalescing Binary Black Holes
}

\author{
Xing-Jiang Zhu ${ }^{1,2}$, E. Howell ${ }^{2}$, T. Regimbau ${ }^{3}$, D. Blair ${ }^{2}$ and Zong-Hong Zhu ${ }^{1}$ \\ ${ }^{1}$ Department of Astronomy, Beijing Normal University, Beijing 100875, China; \\ zhuzh@bnu.edu.cn \\ ${ }^{2}$ School of Physics, University of Western Australia, Crawley WA 6009, Australia \\ ${ }^{3}$ UMR ARTEMIS, CNRS, University of Nice Sophia-Antipolis, Observatoire de la Côte \\ d'Azur, BP 4229, 06304, Nice Cedex 4, France
}

\begin{abstract}
We estimate the stochastic gravitational wave (GW) background signal from the field population of coalescing binary stellar mass black holes (BHs) throughout the Universe. This study is motivated by recent observations of BH-WolfRayet star systems and by new estimates in the metallicity abundances of star forming galaxies that imply $\mathrm{BH}-\mathrm{BH}$ systems are more common than previously assumed. Using recent analytical results of the inspiral-merger-ringdown waveforms for coalescing binary BH systems, we estimate the resulting stochastic GW background signal. Assuming average quantities for the single source energy emissions, we explore the parameter space of chirp mass and local rate density required for detection by advanced and third generation interferometric GW detectors. For an average chirp mass of $8.7 M_{\odot}$, we find that detection through 3 years of cross-correlation by two advanced detectors will require a rate density, $r_{0} \geq 0.5 \mathrm{Mpc}^{-3} \mathrm{Myr}^{-1}$. Combining data from multiple pairs of detectors can reduce this limit by up to $40 \%$. Investigating the full parameter space we find that detection could be achieved at rates $r_{0} \sim 0.1 \mathrm{Mpc}^{-3} \mathrm{Myr}^{-1}$ for populations of coalescing binary BH systems with average chirp masses of $\sim 15 M_{\odot}$ which are predicted by recent studies of BH-Wolf-Rayet star systems. While this scenario is at the high end of theoretical estimates, cross-correlation of data by two Einstein Telescopes could detect this signal under the condition $r_{0} \geq 10^{-3} \mathrm{Mpc}^{-3} \mathrm{Myr}^{-1}$. Such a signal could potentially mask a primordial GW background signal of dimensionless energy density, $\Omega_{\mathrm{GW}} \sim 10^{-10}$, around the $(1-500) \mathrm{Hz}$ frequency range.
\end{abstract}

Subject headings: gravitational waves - binaries: close - cosmology: miscellaneous 


\section{Introduction}

Coalescing systems of stellar mass binary black holes (BBHs) are among the most likely candidates for the first detection of gravitational waves (GWs) (Flanagan \& Hughes 1998; Buonanno et al. 2003). Their enormous predicted luminosities $\sim 10^{23} L_{\odot}$, would allow future ground-based interferometric GW detectors, such as Advanced LIGO (Harry et al. 2010) and Advanced Virgo (Acernese et al.|2009) or third generation instruments such as the Einstein Telescope (ET; Punturo et al. 2010), to probe these sources out to Gpc distances. In this paper, we are motivated by recent increased rate estimates (Belczynski et al. 2010b) to explore the possibility that a population of BBHs could form a detectable stochastic GW background (SGWB) signal for these instruments.

SGWBs can result from the superposition of populations of unresolved primordial (Grishchuk 1974) or astrophysical sources (see Regimbau 2011, for a recent review). Astrophysical SGWB signals are important for at least two reasons. Firstly, they contain rich information on the global properties of source populations, such as their source rate evolution, their mass ranges and their average energy emissions. Secondly, a dominant continuous astrophysical background could mask the relic SGWB signal from the very early Universe (see Maggiore 2000; Buonanno 2003, for reviews).

Mergers of binary neutron stars have been suggested as sources of potentially detectable SGWBs (Regimbau \& de Freitas Pacheco 2006; Regimbau \& Chauvineau 2007). Recent observations of BH-Wolf-Rayet (WR) star systems (Crowther et al. 2010) and new estimates in the metallicity abundances of star forming galaxies (Panter et al. 2008) imply that the Galactic merger rate of BBHs may be of a similar order to that of binary neutron stars (Belczynski et al. 2010b). Therefore, a population of more luminous BBHs could produce a dominant background signal. Our aim is to explore upper limits for a SGWB from coalescing $\mathrm{BBHs}$ over a range of rates and system masses. We investigate the constraints future ground based interferometric GW detectors will be able to place on the average properties of the BBH population.

The paper is organized as follows. In Section 2 we discuss rate estimates of coalescing $\mathrm{BBHs}$ and then derive cosmic source rate evolution models for different star formation histories and minimal delay times. In Section 3, source energy spectra for coalescing BBHs are

obtained using the template gravitational waveforms of Ajith et al. (2008) and Ajith et al. (2009). We then calculate the BBH background in Section 4 and discuss the detection regimes, detectability and constraints on the parameter space of the predicted background in Section 5. Finally, in Section 6 we present our conclusions. 


\section{Rates of BBH coalescences}

The discovery of BH-WR star systems within close proximity has increased rate estimates of coalescing BBHs. There are presently two known systems: NGC300 X-1, which lies at a distance of $1.8 \mathrm{Mpc}$ and is composed of a $\sim 20 M_{\odot} \mathrm{BH}$ and a WR star of $\sim 26 M_{\odot}$ (Crowther et al. 2010); IC10 X-1 contains a BH of a mass at least $23 M_{\odot}$ and a $\sim 35 M_{\odot} \mathrm{WR}$ star, and lies within $700 \mathrm{kpc}$ (Prestwich et al. 2007). As WR stars are the progenitors of Type Ib/c supernovae, if such systems survive the supernova explosion, BBH systems will form and eventually coalesce within a timescale of Gyrs (Bulik et al. 2011).

Recent results from Sloan Digital Sky Survey, have indicated that half of recent star formation involved galaxies with low metallicity (Panter et al. 2008). This has a profound effect on the coalescence rates of compact binaries containing BHs when one considers that NGC300 X-1 and IC10 X-1 were both formed in low metallicity environments.

Survival of BBH systems is highly dependent on whether they can overcome two key obstacles in their evolution. Firstly, post natal supernova kicks can disrupt a significant proportion of systems. Secondly, orbital shrinkage during the common envelope phase when the larger star transfers mass to its smaller companion, can cause the stars to merge before they become compact objects. Belczynski et al. (2010b) have shown through population-synthesis modeling that a lower metallicity environment can suppress these two effects. Firstly, observational evidence suggests that larger BH masses, which are produced at low metallicity, are born with lower kick velocities (Mirabel \& Rodrigues 2003; Belczynski et al. 2010a). Secondly, in lower metallicity environments, slower radial expansion occurs during the common envelope phase, thus increasing binary retention. The greater fraction of systems that can survive, in combination with a greater detection range from more massive and hence luminous systems, has increased the detection prospects of BBHs for ground-based interferometric GW detectors (Belczynski et al. 2010b).

Previous estimates of the coalescence rate of BBHs formed through isolated binary evolution in the field have ranged over orders of magnitude, from $10^{-4}$ to $0.3 \mathrm{Mpc}^{-3} \mathrm{Myr}^{-1}$ with a realistic value of $5 \times 10^{-3} \mathrm{Mpc}^{-3} \mathrm{Myr}^{-1}$ (Kalogera et al. 2007; Abadie et al. 2010). The effect of metallicity discussed above increases the realistic estimate to $3.1 \times 10^{-2} \mathrm{Mpc}^{-3} \mathrm{Myr}^{-1}$ assuming a 50-50 mixture of solar and 10\% solar metallicity and the most stringent evolu-

tionary scenario with respect to system survival (Belczynski et al. 2010b). Unless we state otherwise, this rate (denoted as $r_{1}$ ) is adopted in our calculations.

A higher estimate of $0.43 \mathrm{Mpc}^{-3} \mathrm{Myr}^{-1}$ (denoted as $r_{2}$ ) was obtained by assuming that all systems survive early merger during the common envelope stage. We note that $r_{2}$ leads to a detection rate for initial LIGO/Virgo of around 5 events per year. A recent population 
synthesis study by Bulik et al. (2011), however, has demonstrated the formation of BBHs with high chirp masses $\left(\sim 15 M_{\odot}\right)$ from the two BH-WR systems, and has yielded a similarly

high rate of $0.36 \mathrm{Mpc}^{-3} \mathrm{Myr}^{-1}$ corresponding to 3.6 detections a year. They suggest that either currently employed searches are insensitive to higher mass BBH inspirals or that there is an additional aspect to the evolution of such systems that has not so far been considered. To take account of this uncertainty, we take $r_{2}$ as a higher rate.

\subsection{The cosmic rate evolution model}

The cosmic coalescence rate can be extrapolated from the local rate density $r_{0}$ by assuming the rate tracks the star formation rate (SFR). Explicitly, the differential GW event rate in the redshift shell $z$ to $z+d z$ can be written as

$$
d R=r_{0} e(z) \frac{d V}{d z} d z
$$

with $d V$ the cosmology dependent co-moving volume element given by

$$
\frac{d V}{d z}=4 \pi c \frac{r(z)^{2}}{H(z)}
$$

where the Hubble parameter $H(z)=H_{0}\left[\Omega_{\Lambda}+\Omega_{m}(1+z)^{3}\right]^{1 / 2}$ and $r(z)$ is the comoving distance related to the luminosity distance by $d_{L}=r(1+z)$. We use the parameters $H_{0}=$ $100 h \cdot \mathrm{km} \mathrm{s}^{-1} \mathrm{Mpc}^{-1}$ with $h=0.7, \Omega_{m}=0.3$ and $\Omega_{\Lambda}=0.7$ (Komatsu et al. 2009).

Source rate density evolution is accounted for by the dimensionless evolution factor $e(z)$, normalized to unity in our local intergalactic neighbourhood. Following Regimbau \& Hughes (2009), we define $e(z)=\dot{\rho}_{*, c}(z) / \dot{\rho}_{*, c}(0)$ normalized to unity at $z=0$ where

$$
\dot{\rho}_{*, c}(z)=\int \frac{\dot{\rho}_{*}\left(z_{f}\right)}{\left(1+z_{f}\right)} P\left(t_{d}\right) d t_{d}
$$

relates the SFR to the $\mathrm{BBH}$ coalescence rate. Here, $\dot{\rho}_{*}$ is the SFR density in $M_{\odot} \mathrm{yr}^{-1} \mathrm{Mpc}^{-3}$, based on the parametric form of Hopkins \& Beacom (2006), derived from recent measurements of the galaxy luminosity function. To allow for uncertainties in the SFR, we also consider a recent model described in Wilkins et al. (2008), obtained through measurements of the stellar mass density - this model gives a much lower rate for $z>1$. The factors $z$ and $z_{f}$ represent the redshift values of $\mathrm{BBH}$ merger and $\mathrm{BBH}$ system formation respectively. The delay time for BBHs, $t_{d}$, is given by the difference in lookback times between $z_{f}$ and $z$ 


$$
t_{d}=\int_{z}^{z_{f}} \frac{d z^{\prime}}{\left(1+z^{\prime}\right) H\left(z^{\prime}\right)}
$$

Here $P\left(t_{d}\right)$ is the probability distribution of delay times. For this we take the form, $1 / t_{d}$, with a lower cutoff $t_{0}$ at $100 \mathrm{Myr}$ as suggested by current population synthesis studies (Belczynski et al. 2002; Postnov \& Yungelson 2006; Dominik et al. 2011). To account for uncertainty in the lower cutoff and possible correlation between $P\left(t_{d}\right)$ and BBH chirp masses, we additionally consider a longer minimal delay time of $t_{0}=500 \mathrm{Myr}$ ( T. Bulik, private communication).

Figure 1. shows the cosmic rate evolution factor $e(z)$ of $\mathrm{BBH}$ coalescences for different SFRs and minimal delay times. Although the models show some variation in peak $z$, we show in section 4 that the magnitude of the background is largely dependent on $r_{0}$ rather than the form of $e(z)$.

\section{The GW energy spectrum of coalescing BBHs}

The evolution of a coalescing $\mathrm{BBH}$ is traditionally divided into three phases: inspiral, merger and ringdown. While the early inspiral and ringdown phases can be approximated analytically by post-Newtonian expansion and perturbation theory, to model the late inspiral and merger requires a numerical solution of the Einstein equations. In the last few years breakthroughs in numerical relativity have enabled the inspiral-merger-ringdown evolution of $\mathrm{BBH}$ coalescences to be modeled with high accuracy for a broad space of parameters (see, e.g., Hannam 2009; Hinder 2010, for reviews).

For the complete evolution history of coalescing BBHs, phenomenological waveforms can be constructed by frequency domain matching of post-Newtonian inspiral waveforms with coalescence waveforms from numerical simulations (Ajith et al. 2007; Buonanno et al. 2007; Pan et al. 2008; Santamaría et al. 2010). Such waveforms share a common feature, in that the Fourier amplitude is approximated to a leading order as a power-law function of frequency $\nu^{-7 / 6}$ for the inspiral phase, followed by $\nu^{-2 / 3}$ for the merger stage and a Lorentzian function around the quasi-normal mode ringdown frequency for the ringdown stage.

For this study, we convert to a energy spectrum to account for the individual source emissions. We choose two template models: one from Ajith et al. (2008), for a non-spinning case, and another from Ajith et al. (2009) for BBHs with non-precessing spins. In Ajith et al. (2008) the Fourier amplitude is given by their equation (4.13), which we convert to an energy spectrum $d E / d \nu$. As we expect the inspiral spectrum to equal the post-Newtonian approx- 
imation (see, e.g., Cutler et al. 1993; Finn \& Chernoff 1993), the inspiral-merger-ringdown spectrum for a $\mathrm{BBH}$ with component masses $m_{1}$ and $m_{2}$ is given by

$$
\frac{d E}{d \nu} \equiv \frac{(G \pi)^{2 / 3} M_{c}^{5 / 3}}{3}\left\{\begin{array}{l}
\nu^{-1 / 3} \text { if } \nu<\nu_{1} \\
\omega_{1} \nu^{2 / 3} \text { if } \nu_{1} \leq \nu<\nu_{2} \\
\omega_{2}\left[\frac{\nu}{1+\left(\frac{\nu-\nu_{2}}{\sigma / 2}\right)^{2}}\right]^{2} \text { if } \nu_{2} \leq \nu<\nu_{3}
\end{array}\right.
$$

Here $M_{c}$ is the chirp mass, $M_{c}^{5 / 3}=m_{1} m_{2}\left(m_{1}+m_{2}\right)^{-1 / 3}, \omega_{1}=\nu_{1}^{-1}$ and $\omega_{2}=\nu_{1}^{-1} \nu_{2}^{-4 / 3}$ are constants chosen to make $d E / d \nu$ continuous across $\nu_{1}$ and $\nu_{2}$. The set of parameters $\left(\nu_{1}, \nu_{2}, \sigma, \nu_{3}\right)$ can be determined by the two physical parameters (the total mass $M$ and the symmetric mass ratio $\eta)$ in terms of $\left(a \eta^{2}+b \eta+c\right) / \pi M$, with coefficients $a, b, c$ given in Table 1 of Ajith et al. (2008), producing $(404,807,237,1153) \mathrm{Hz}$ for a $10 M_{\odot}-10 M_{\odot} \mathrm{BBH}$ $\left(M_{c}=8.7 M_{\odot}\right)$.

The waveform presented in Ajith et al. (2009), includes spin effects through a single spin parameter $\chi=(1+\delta) \chi_{1} / 2+(1-\delta) \chi_{2} / 2$, with $\delta=\left(m_{1}-m_{2}\right) / M$ and $\chi_{i}=S_{i} / m_{i}^{2}$. The parameter $S_{i}$ represents the spin angular momentum of the $i$ th black hole. The corresponding Fourier amplitude includes a minor correction (related to $\chi$ and $\eta$ ) for non-spinning BBHs. We construct energy spectra for BBHs with non-precessing spins based on their equation (1).

Figure 2 shows the GW energy spectra for a $10 M_{\odot}-10 M_{\odot} \mathrm{BBH}$ assuming: the nonspinning case; $\chi=0.85 ; \chi=0$ and $\chi=-0.85$. The two extreme values for $\chi$ are set by the numerical simulations of Ajith et al. (2009), corresponding to both binary components having maximal spins aligned or anti-aligned with the orbital angular momentum. The radiation efficiencies for these energy spectra are $6.7 \%, 9.74 \%, 5.15 \%$ and $4.28 \%$ respectively. We note that the radiated GW energy mainly depends on $M_{c}$ and $\chi$, and that the energy spectra for $\nu \lesssim 100 \mathrm{~Hz}$ show little variation. We note that effect of orbital eccentricity is not considered in our derivation of energy spectrum. This has little effect on our results as the orbits of coalescing compact objects are expected to circularise (Peters 1964) before their GW signals reach the sensitive frequency band of ground-based interferometric detectors (Brown \& Zimmerman 2010). 


\section{The stochastic GW background}

In this section we evaluate the spectral properties of the BBH background. It is customary to characterize the SGWB by the energy density parameter

$$
\Omega_{\mathrm{GW}}\left(\nu_{\mathrm{obs}}\right)=\frac{1}{\rho_{c}} \frac{d \rho_{\mathrm{GW}}}{d \ln \nu_{\mathrm{obs}}},
$$

where $\rho_{\mathrm{GW}}$ is the GW energy density, $\nu_{\mathrm{obs}}$ is the observed GW frequency and $\rho_{c}=3 H_{0}^{2} / 8 \pi G$ is the present value of critical energy density required to close the Universe. For a SGWB of astrophysical origin, $\Omega_{\mathrm{GW}}$ is related to the spectral energy density $F_{\nu}$ (in $\operatorname{erg~cm}^{-2} \mathrm{~Hz}^{-1} \mathrm{~s}^{-1}$ ) by

$$
\Omega_{\mathrm{GW}}\left(\nu_{\mathrm{obs}}\right)=\frac{\nu_{\mathrm{obs}}}{c^{3} \rho_{c}} F_{\nu}\left(\nu_{\mathrm{obs}}\right),
$$

where $F_{\nu}$ at the observed frequency $\nu_{\text {obs }}$ can be written as

$$
F_{\nu}\left(\nu_{\mathrm{obs}}\right)=\int_{0}^{z_{\mathrm{sup}}} f_{\nu}\left(\nu_{\mathrm{obs}}, z\right) \frac{d R}{d z}(z) d z,
$$

Here, $z_{\text {sup }}=\min \left(z_{\max }, \nu_{\max } / \nu_{\text {obs }}-1\right)$, with $z_{\max }$ the maximum redshift of SFR model and $\nu_{\max }$ the maximal emitting GW frequency. The differential GW event rate, $d R / d z$, is given by equation (1) and $f_{\nu}$ is the energy flux per unit frequency emitted by a source at a luminosity distance $d_{L}(z)$

$$
f_{\nu}\left(\nu_{\mathrm{obs}}, z\right)=\frac{1}{4 \pi d_{L}(z)^{2}} \frac{d E}{d \nu}(1+z),
$$

where $d E / d \nu$ is the gravitational energy spectrum given by equation (5) and $\nu$ is the frequency in the source frame which is related to the observed frequency by $\nu=\nu_{\mathrm{obs}}(1+z)$.

Figure 3 shows the energy density parameter $\Omega_{\mathrm{GW}}$ of the $\mathrm{BBH}$ background corresponding to five models outlined in Table 1 . The final model (e) approximates the common powerlaw behavior of models (a-d) before reaching a peak of $\sim 10^{-9}$ at $400-600 \mathrm{~Hz}$, and uses a conservative estimate to account for effects of spin, SFR and delay time. This model is given by $\Omega_{\mathrm{GW}}\left(\nu_{\mathrm{obs}}\right)=1.95 \times 10^{-11} \nu_{\mathrm{obs}}^{2 / 3}$ for $1 \mathrm{~Hz}<\nu_{\mathrm{obs}}<400 \mathrm{~Hz}$. We note that using the two extreme spectra $(\chi=0.85$ and $\chi=-0.85)$ in Figure 2 leads to a variation in the results of less than $40 \%$. 
We see in Figure 3 that effects of spin, SFR and delay time are insignificant for estimating the $\mathrm{BBH}$ background considering all other uncertainties in sources rates and systems masses. Combining the equations used to calculate $\Omega_{\mathrm{GW}}$, we find the $\mathrm{BBH}$ background is sensitive to $r_{0}$ and average value of $M_{c}$ through the relation $\Omega_{\mathrm{GW}} \propto r_{0} M_{c}^{5 / 3}$. It is useful to express model (e) in the form:

$$
\Omega_{\mathrm{GW}}\left(\nu_{\mathrm{obs}}\right) \simeq 4.2 \times 10^{-10}\left(\frac{r_{0}}{3.1 \times 10^{-2} \mathrm{Mpc}^{-3} \mathrm{Myr}^{-1}}\right)\left(\frac{M_{c}}{8.7 M_{\odot}}\right)^{5 / 3}\left(\frac{\nu_{\mathrm{obs}}}{100 \mathrm{~Hz}}\right)^{2 / 3}
$$

allowing us investigate the parameter space $\left(r_{0}, M_{c}\right)$ for a SGWB in the next section. We note that equation (10) is consistent with equation (10) of Phinney (2001). Considering that the most sensitive frequency regime of the second and third generation detectors is within 100-200 Hz, we further adopt a cutoff frequency of $400 \mathrm{~Hz}$ when applying this function.

\section{Issues of detectability}

A number of laser interferometric GW detectors have reached their design sensitivities and have been coordinating as a global array. These include the LIGO1 detectors based at Hanford (H) and Livingston (L) in USA, the Virgd2 (V) detector in Italy and the GEO6003 detector in Germany. The LIGO and Virgo detectors are undergoing upgrades that will produce a order of magnitude improvement in sensitivity. Advanced LIGO is expected to

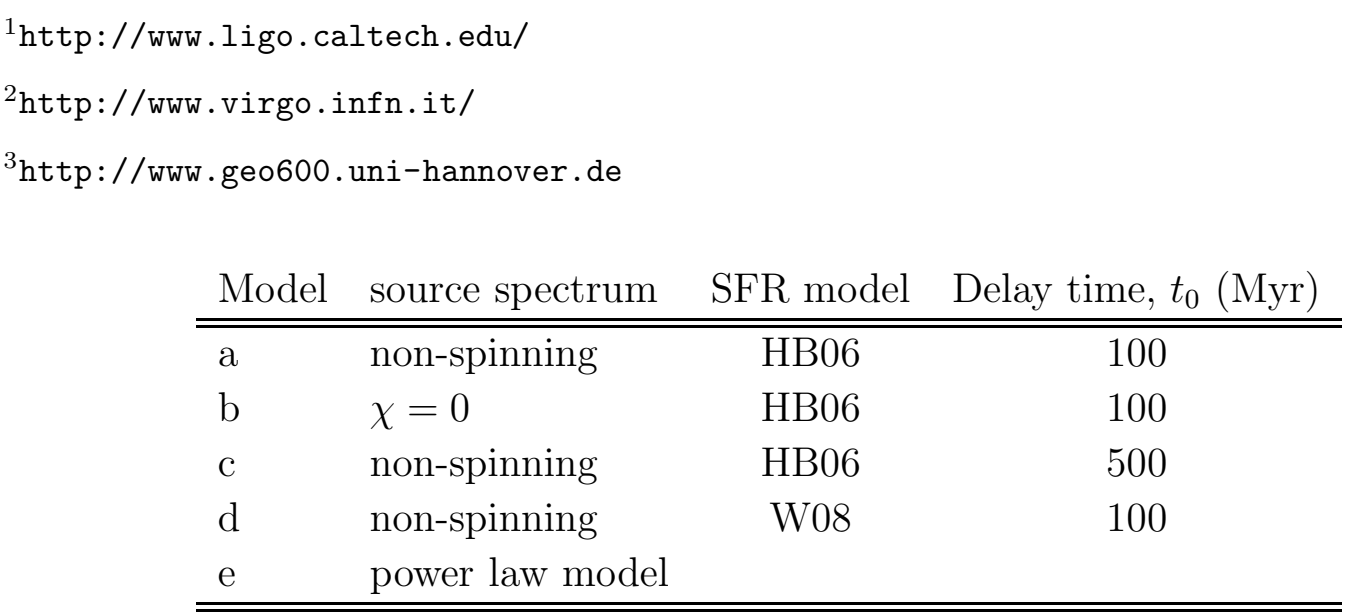

Table 1: The 5 models and their parameters used to determine the energy density parameter $\Omega_{\mathrm{GW}}$ of a $\mathrm{BBH}$ background. Model e, a power law approximation, is described in the text. 
be operational by 2015 and an Advanced Virgo facility is expected to begin commissioning in 2011. Other plans for advanced detectors include LCGT 4 (the Large-scale Cryogenic Gravitational-wave Telescope; denoted as C), an underground detector to be built by the TAMA group in Japan and LIGO-Australia 5 (A), a 4km interferometric detector that would be based in Western Australia. Third generation detectors have also been proposed, such as ET, with a target sensitivity 100 times greater than current instruments.

To evaluate the detectability of the BBH background, we consider the five advanced detectors introduced above $(\mathrm{H}, \mathrm{L}, \mathrm{V}, \mathrm{C}, \mathrm{A})$. We take LIGO-Australia to have the same sensitivity as Advanced LIGO and for ET adopt ET-B sensitivity from Hild et al. (2008) as well as the latest ET-D sensitivity from Hild et al. (2011). Design sensitivity curves 6 for these detectors are shown in Figure 4.

\subsection{Duty Cycle}

For a SGWB of astrophysical origin, in addition to the energy density parameter and characteristic frequency, another useful quantity is the duty cycle $(D C)$. This is defined as the ratio of the typical duration of a single signal to the average time interval between successive events, e.g.,

$$
D C=\int_{0}^{z_{\max }} \bar{\tau}(z) d R(z)
$$

where $d R(z)$ is the differential event rate given by equation (1) and $z_{\max }$ corresponds to the redshift limit of considered SFR models. $\bar{\tau}(z)$ is the average observed duration of GW signals generated by individual sources at redshift $z$, given at leading order by

$$
\bar{\tau}(z)=\frac{5 c^{5}}{256 \pi^{8 / 3} G^{5 / 3}}\left[(1+z) M_{c}\right]^{-5 / 3} \nu_{\min }^{-8 / 3}
$$

Here, $\nu_{\min }$ is the lower frequency bound of the detector, which is set by the low-frequency seismic "wall" for ground-based interferometric GW detectors. The present LIGO detector has $\nu_{\min } \simeq 40 \mathrm{~Hz}$, and this can be reduced to $10 \mathrm{~Hz}$ for advanced detectors and $1 \mathrm{~Hz}$ for ET (see Regimbau \& Hughes 2009, for details).

\footnotetext{
${ }^{4}$ http://gw.icrr.u-tokyo.ac.jp/lcgt/

${ }^{5}$ http://www.aigo.org.au/

${ }^{6}$ Data are taken from http://wwwcascina.virgo.infn.it/advirgo/, https://dcc.ligo.org/cgibin/DocDB/ShowDocument?docid=2974, http://www.et-gw.eu/etsensitivities and Kuroda et al. (2010).
} 
In general, a GW background with a $D C$ of unity or above is defined as continuous 7 . And non-continuous signals can be further categorized into popcorn noise $(0.1 \leq D C<1)$ and shot noise $(D C<0.1)$ type. As source rate evolution will increase out to large cosmological volumes, some studies investigate how $D C$ too increases with $z$ (Coward \& Regimbau 2006; Howell et al. 2011). In this study we are concerned with the value of $D C$ as seen at the detector and thus equation (11) is interpreted as a total value.

For a background signal produced by a source population with an average chirp mass $\left\langle M_{c}\right\rangle=8.7 M_{\odot}$, for rates $r_{1}$ and $r_{2}$ we find $D C$ values of 0.01 and 0.2 for advanced detectors. Therefore the signal will most likely be of the shot noise category (or at most popcorn noise for the higher rate $r_{2}$ ). For ET type detectors the signal will be continuous with $D C$ values of 5.8 and 80.6 for rates $r_{1}$ and $r_{2}$ respectively.

Although for advanced detectors, the SGWB calculated in this paper is not continuous (Gaussian) even at the higher rate $r_{2}$, we note that Drasco \& Flanagan (2003) have found the cross correlation method nearly optimal for a $D C>10^{-3}$ over 1-year integration 8 . In the following sections we therefore consider the cross correlation statistic to assess the detectability of the estimated BBH background.

\subsection{Cross correlation of detectors}

The optimum detection strategy for continuous GW background signals is cross-correlating the output of two neighbouring detectors (see, Allen \& Romano 1999; Maggiore 2000). This requires that the detectors are separated by less than one reduced wavelength, which is about $100 \mathrm{~km}$ for frequencies around $500 \mathrm{~Hz}$ where $\Omega_{\mathrm{GW}}(f)$ might peak. The detectors also need to be sufficiently well separated that their noise sources are largely uncorrelated. We note that although this may not be possible for ET, techniques are in development to remove environmental noise and instrumental correlations (Fotopoulos 2008).

Under these conditions, assuming Gaussian noise in each detector and optimal filtering, a filter function chosen to maximize the signal-to-noise ratio, SNR for two such detectors is

\footnotetext{
${ }^{7}$ We note that to allow for a small number of events that may be resolved at $D C \sim 1$, some authors consider a more conservative threshold of $D C \sim 10$ to indicate the continuous regime (Regimbau \& Hughes 2009; Howell et al. 2011).

${ }^{8}$ We note however, Drasco \& Flanagan (2003) also proposed a new statistic to search for SGWB with low duty cycles, which is currently being investigated in the LIGO/Virgo collaboration.
} 
given by (Allen \& Romano 1999, equation 3.75)

$$
\mathrm{SNR}^{2}=\frac{9 H_{0}^{4}}{50 \pi^{4}} T \int_{0}^{\infty} d f \frac{\gamma^{2}(f) \Omega_{\mathrm{GW}}^{2}(f)}{f^{6} P_{1}(f) P_{2}(f)}
$$

Here $\gamma(f)$ is the 'overlap reduction function', which accounts for the separation and relative orientation of the detectors (Flanagan 1993), and $P_{1}(f)$ and $P_{2}(f)$ are the noise power spectral densities of the detectors, and $T$ is the integration time. As the optimal filter depends on $\Omega_{\mathrm{GW}}(f)$, a range of filter functions based on theoretical expectations of this function will need to be used.

In this study we use data of relative positions and orientations for 10 independent pairs of the five advanced detectors given in Table 3 of Nishizawa et al. (2009) and employ the tensor-mode functions described in their equations (33-35). For ET we assume two detectors of triangular shape $\left(60^{\circ}\right.$ between the two arms) and separated by an angle of $120^{\circ}$, for which the $\gamma(f)$ has a constant value of $-3 / 8$ from $1 \mathrm{~Hz}$ to $1000 \mathrm{~Hz}$ (Howell et al. 2011). We adopt a value of $\mathrm{SNR}=3$ to indicate detection, corresponding with false alarm rate of $10 \%$ and

detection rate of $90 \%$ (Allen \& Romano 1999). We also assume an integration time of 3 years for advanced detectors and 1 year for ET.

\subsection{Assessing the detectability using the worldwide network}

Calculating the SNRs for SGWB model (e) shown in Figure 3, we find a value of 0.14 through cross-correlation by two the Advanced LIGO detectors H-L. For the other four models shown in Figure 3, we find variation in SNR of within 20\%. For ET we find SNRs of 59 and 112 assuming ET-B and ET-D sensitivities respectively for model (e), indicating that this signal will be easily-detected by third generation detectors. These results, based on average quantities, suggest that to detect the BBH background with two Advanced LIGO detectors will require a rate greater than even the higher rate estimate, $r_{2} \sim 0.43 \mathrm{Mpc}^{-3} \mathrm{Myr}^{-1}$. As there will exist variation in the sensitivities, locations and orientations of detectors within a worldwide detector network, it is useful to compare the performances of different detector pairs and investigate how combining the network could improve the detection prospects.

Two approaches of combining $2 \mathrm{~N}$ detectors to increase the sensitivity of a stochastic background search have been proposed by Allen \& Romano (1999). We apply these two methods to a network of 4 second generation detectors. In each case, the optimal SNR can be expressed as follows, with individual detectors (1-4) indicated in parenthesis:

(i) Four-detector correlation (FC) - can be performed by directly correlating the outputs of 4 detectors 


$$
\mathrm{SNR}_{\mathrm{optI}}^{2} \approx{ }^{(12)} \mathrm{SNR}^{2}{ }^{(34)} \mathrm{SNR}^{2}+{ }^{(13)} \mathrm{SNR}^{2(24)} \mathrm{SNR}^{2}+{ }^{(14)} \mathrm{SNR}^{2}{ }^{(23)} \mathrm{SNR}^{2} .
$$

(ii) Combining multiple pairs $(\mathrm{CP})$ - is performed by correlating the outputs of a pair of detectors, and then combining measurements from multiple detector pairs

$$
\mathrm{SNR}_{\mathrm{optII}}^{2}={ }^{(12)} \mathrm{SNR}^{2}+{ }^{(13)} \mathrm{SNR}^{2}+\cdots+{ }^{(34)} \mathrm{SNR}^{2} .
$$

We now investigate the detectability of the BBH background by considering both these two approaches, as well as the cross-correlation method between two detectors. To do this, we substitute equation (10) for $\Omega_{\mathrm{GW}}$ into equation (13). Although there are uncertainties in $r_{0}$ and the true ranges of $M_{c}$, this approximation allows us to quantify the advantages of the different approaches outlined above. The goal here is to provide insight into both the requirements for a detection and the constraints that can be supported by a null-detection.

Firstly, we set $M_{\mathrm{c}}=8.7 M_{\odot}$ and compare standard cross-correlation measurements between different pairs of the five advanced detectors (H,L,V,C,A). We also determine any improvements that can be gained from the two approaches described above - (i) FC and (ii) CP. We assess performance through the values of $r_{0}$ required to produce a $\mathrm{SNR}=3$.

Table 2 outlines the constraints on $r_{0}$ we obtain through these different approaches for second generation detectors. We see that variation in the function $\gamma(f)$ between different detector pairs together with different sensitivity levels, produces a variation in the values of $r_{0}$ required for detection. Among the 10 independent pairs of the five advanced detectors, $\mathrm{H}-\mathrm{L}$ performs best as indicated by the lowest required value of $r_{0}\left(0.55 \mathrm{Mpc}^{-3} \mathrm{Myr}^{-1}\right)$. We note that these values are above the higher rate $r_{2}$. For cross-correlation measurements between two ETs, minimum detectable values of $r_{0} \sim 1.34 \times 10^{-3} \mathrm{Mpc}^{-3} \mathrm{Myr}^{-1}$ and $r_{0} \sim$ $7 \times 10^{-4} \mathrm{Mpc}^{-3} \mathrm{Myr}^{-1}$ are obtained using ET-B and ET-D sensitivities respectively.

The values presented by Four-detector correlation, FC, suggest that no considerable advantage can be obtained through this approach. However, we see that by combining multiple pairs, CP, produces the most consistent improvement, the best of which comes from the AHLV combination (around a 40\% improvement on H-L). Equation (15) shows that applying the $\mathrm{CP}$ method to the 3 identical arms of ET will reduce the minimum detectable values of $r_{0}$ by a factor of $\sqrt{3}$.

\subsection{The $r_{0}-M_{c}$ parameter space of a detectable BBH background}

We now adopt the same methods as employed in the last section to explore the parameter space of $r_{0}-M_{c}$ required for a detectable BBH background. To allow for uncertainties in $M_{c}$, 
we consider a large range of (4-20) $M_{\odot}$. This range includes most values of $M_{c}$ within the low metallicity $\left(0.1 Z_{\odot}\right)$ distribution of Belczynski et al. (2010b). We note however, that for the more realistic scenario that accounts for early common envelope mergers as the stars pass through the Hertzsprung gap, the range is more constrained, with $M_{c}$ around (4-9) $M_{\odot}$.

Figure 5 shows the $r_{0}-M_{c}$ space for three different detection scenarios: 1) cross correlation of $\mathrm{H}-\mathrm{L}$ - the best performing pair of advanced detectors; 2) AHLV (through CP) - the optimal combination of 4 advanced detectors; 3) cross correlation of two ET type detectors using two possible sensitivities (ET-B and ET-D). Zones encompassed by the two rates, $r_{1}$ and $r_{2}$, are shown by the shaded areas.

The figure further confirms that advanced detectors are not likely to detect the $\mathrm{BBH}$ background at the rate $r_{1}$. Detection would require the high rate estimate, $r_{2}$ and $M_{c} \gtrsim$ $10 M_{\odot}$. Such a range for $r_{0}-M_{c}$ has been predicted by Bulik et al. (2011) through studies of the two BH-WR systems, NGC300 X-1 and IC10 X-1. A null detection would therefore confirm a lack of understanding in the binary evolution of such systems based on the single source models employed in this study.

In regards to detection strategies for SGWB signals, Figure 5 shows some improvement in detectability through the use of CP. As already noted in Table 2, AHLV can improve the detectability by $40 \%$ compared with only two Advanced LIGO detectors (H-L).

For ET, for the entire range of $M_{c}$ the signal would be comfortably detected at the lower rate $r_{1}$. A detectable continuous background requires a rate of order $10^{-3} \mathrm{Mpc}^{-3} \mathrm{Myr}^{-1}$ or above. In comparison with ET-B, we find that a larger parameter space can be probed by ET-D due to an improved sensitivity at lower frequencies $(<20 \mathrm{~Hz})$.

\section{Conclusions}

In this paper we have estimated the potential contribution of a population of coalescing BBHs to a SGWB signal. We are motivated by recent observations of BH-WR star systems (Crowther et al. 2010) and by new estimates in the metallicity abundances of star forming galaxies (Panter et al. 2008) that have suggested the rate of BBHs in field populations may be greater than previously expected. We base the single source emissions on energy spectra calculated from recent parameterized waveforms of Ajith et al. (2008, 2009). Then, assuming that the $\mathrm{BBH}$ rate traces the SFR with some delay time, we derive cosmic source rate evolution models and extrapolate our single-source model out to high redshifts. Rather than a population synthesis approach, we assume average properties (e.g., masses and spins) 
for the $\mathrm{BBH}$ population to determine the characteristics of the SGWB signal and principal parameters to which the BBH background is sensitive.

Our results show that for $M_{c} \lesssim 10 M_{\odot}$, the background is not likely to be detected through cross-correlation by two advanced detectors even at $r_{2} \sim 0.43 \mathrm{Mpc}^{-3} \mathrm{Myr}^{-1}$. Only for greater values of $M_{c}$, as have been predicted to result from BH-WR systems such as NGC300 X-1 and IC10 X-1 (Bulik et al. 2011), there is scope for detection.

To further assess the detection prospects for second generation detectors, we have considered the possibility of combining a worldwide network of advanced detectors to improve the cross-correlation statistic, namely the methods FC and CP described in section 5.3. We find that of these two approaches, CP can produce an improvement of up to $40 \%$ against a standard cross-correlation between two detectors. For the third generation detector, ET, the signal is accessible with a SNR of 59 and 112 at the lower rate estimate $r_{1} \sim 3.1 \times 10^{-2} \mathrm{Mpc}^{-3} \mathrm{Myr}^{-1}$ using ET-B and ET-D sensitivities respectively. This signal could mask the primordial background signal at below around $\Omega_{\mathrm{GW}} \sim 4 \times 10^{-10}$ at $\sim 100 \mathrm{~Hz}$.

We note that the rates used in this study are computed assuming Milky Way type galaxies and the standard formation channel - isolated binary evolution. Massive binary formation in early elliptical galaxies is expected to improve the coalescence rate (de Freitas Pacheco et al. 2006; O'Shaughnessy et al. 2010). This is particularly important for BBHs due to the longer delay time. In addition, dynamical formation scenarios in dense stellar environments can make a significant contribution to BBH rates. These other formation channels will not only increase the event rate of coalescing $\mathrm{BBHs}$, but also add additional uncertainty to the average component masses of the BBH population. For example, simulations by Sadowski et al. (2008) suggest that the average chirp mass in clusters is $\left\langle M_{\mathrm{c}}\right\rangle \sim 20 M_{\odot}$, much larger than the same found in the field $\left\langle M_{\mathrm{c}}\right\rangle \sim 7 M_{\odot}$. This might indicate two similar backgrounds peaking at quite different frequencies. We have shown a minimum detectable $r_{0}$ for ET at around $10^{-3} \mathrm{Mpc}^{-3} \mathrm{Myr}^{-1}$, which may be in the range of rate predictions from dynamical formation scenarios (O'Leary et al. 2007; Sadowski et al. 2008; Miller \& Lauburg 2009). Therefore, detection at the sensitivity of ET could enable these two potential background signals to be untangled, thus allowing the average properties of the different populations to be probed.

Additionally, clues to how these two populations contribute to a confusion background may be provided by Advanced LIGO/Virgo through single detections in the shot noise $(D C \ll 0.1)$ regime. New data analysis techniques, such as the probability event horizon method (Coward \& Burman 2005; Howell et al. 2007) which extracts the temporal signature from a population of transient sources or the maximum likelihood statistic (Drasco \& Flanagan 2003 ), could prove valuable in interrogating this regime. 
In view of future searches for a primordial background signal, particularly for ET, simulations incorporating both dynamical and isolated binary formation channels could prove useful. These will be considered in a future study.

\section{Acknowledgments}

We thank Cole Miller for useful discussions during the early stages of this study. We also thank Luciano Rezzolla for a careful reading of the initial manuscript and for providing important feedback on the gravitational wave waveform parameters of coalescing BBHs. The authors are also grateful to Tomasz Bulik for insightful comments which have led to some valuable amendments and for providing plots of chirp mass-delay time relation, to Krzysztof Belczynski and Michal Dominik for providing the distribution of the BBH delay time from StarTrack simulations, and to Ilya Mandel for useful discussion about the BBH parameters. We thank the anonymous referee for useful suggestions which improved the clarity and presentation of our results. Z.X.-J. acknowledges the Australian Research Council and the W.A. Government Center of Excellence Programme for support of his visit at UWA where this work had been done. Z.Z.-H. is supported by the National Science Foundation of China

under the Distinguished Young Scholar Grant 10825313 and by the Ministry of Science and Technology national basic science Programme (Project 973) under grant No. 2007CB815401.

\section{REFERENCES}

Abadie, J., et al. 2010, Class. Quantum Grav., 27, 173001

Acernese, F., et al. 2009, Advanced Virgo baseline design, Virgo Internal Note VIR-0027A-09 (https://pub3.ego-gw.it/itf/tds/file.php?callFile=VIR-0027A-09.pdf)

Ajith, P., et al. 2007, Class. Quantum Grav., 24, S689

Ajith, P., et al. 2008, Phys. Rev. D, 77, 104017

Ajith, P., et al. 2009, arXiv:0909.2867

Allen, B., \& Romano, J. D. 1999, Phys. Rev. D, 59, 102001

Belczynski, K., Bulik, T., Fryer, C. L., Ruiter, A., Valsecchi, F., Vink, J. S., \& Hurley, J. R. 2010a, ApJ, 714, 1217

Belczynski, K., Dominik, M., Bulik, T., O’Shaughnessy, R., Fryer, C., Holz, D. E. 2010b, ApJ, 715, L138 
Belczynski, K., Kalogera, V., \& Bulik, T. 2002, ApJ, 572, 407

Brown, D. A. and Zimmerman, P. J. 2010, Phys. Rev. D., 81, 024007

Bulik, T., Belczynski, K., \& Prestwich, A. 2011, ApJ, 730, 140

Buonanno, A. 2003, arXiv:gr-qc/0303085

Buonanno, A., Chen, Y., \& Vallisneri, M. 2003, Phys. Rev. D, 67, 024016

Buonanno, A., Pan, Y., Baker, J. G., Centrella, J., Kelly, B. J., McWilliams, S. T., \& van Meter, J. R. 2007, Phys. Rev. D, 76, 104049

Coward, D. M., \& Burman, R. R. 2005, MNRAS, 361, 362

Coward D., Regimbau T., 2006, New Astron. Rev., 50, 461

Crowther, P. A., Barnard, R., Carpano, S., Clark, J. S., Dhillon, V. S., \& Pollock, A. M. T. 2010, MNRAS, 403, L41

Cutler, C., et al. 1993, Phys. Rev. Lett., 70, 2984

de Freitas Pacheco, J. A., Regimbau, T., Spallici, A., \& Vincent, S. 2006, Int. J. Mod. Phys. D, 15,235

Dominik, M., et al. 2011, in preparation.

Drasco, S., \& Flanagan, É. É. 2003, Phys. Rev. D, 67, 082003

Finn, L. S., \& Chernoff, D. F. 1993, Phys. Rev. D, 47, 2198

Flanagan, E. 1993, Phys. Rev. D, 48, 2389

Flanagan, É. É., \& Hughes, S. A. 1998, Phys. Rev. D, 57, 4535

Fotopoulos, N. V. 2008, J. Phys.: Conf. Ser., 122, 012032

Grishchuk, L. 1974, Soviet Journal of Experimental and Theoretical Physics, 40, 409

Hannam, M. 2009, Class. Quantum Grav., 26, 114001

Harry, G. M., et al. 2010, Class. Quantum Grav., 27, 084006

Hild, S., et al. 2011, Class. Quantum Grav., 28, 094013

Hild, S., Chelkowski, S., \& Freise, A. 2008, arXiv:0810.0604 
Hinder, I. 2010, Class. Quantum Grav., 27, 114004

Hopkins, A. M., \& Beacom, J. F. 2006, ApJ, 651, 142

Howell, E., Coward, D., Burman, R., \& Blair, D. 2007, MNRAS, 377, 719

Howell, E., Regimbau, T., Corsi, A., Coward, D., \& Burman, R. 2011, MNRAS, 410, 2123

Kalogera, V., Belczynski, K., Kim, C., O’Shaughnessy, R., \& Willems, B. 2007, Phys. Rep., 442,75

Komatsu, E., et al. 2009, ApJS, 180, 330

Kuroda, K., et al. 2010, Class. Quantum Grav., 27, 084004

Maggiore, M. 2000, Phys. Rep., 331, 283

Miller, M. C., \& Lauburg, V. M. 2009, ApJ, 692, 917

Mirabel, I. F., \& Rodrigues, I. 2003, Science, 300, 1119

Nishizawa, A., Taruya, A., Hayama, K., Kawamura, S., \& Sakagami, M.-A. 2009, Phys. Rev. D, 79, 08200

O’Leary, R., O’Shaughnessy, R., \& Rasio F. 2007, Phys. Rev. D, 76, 061504

O’Shaughnessy, R., Kalogera, V., \& Belczynski, K. 2010, ApJ, 716, 615

Pan, Y., et al. 2008, Phys. Rev. D, 77, 024014

Panter, B., Jimenez, R., Heavens, A. F., \& Charlot, S. 2008, MNRAS, 391, 1117

Peters, P. C. 1964, Phys. Rev., 136, B1224

Phinney, E. S. 2001, arXiv:astro-ph/0108028

Postnov, K. A., \& Yungelson, L. R. 2006, Living Reviews in Relativity, 9, 6

Prestwich, A. H., et al. 2007, ApJ, 669, L21

Punturo, M., et al. 2010, Class. Quantum Grav., 27, 194002

Regimbau, T. 2011, Res. Astron. Astrophys., 11, 369

Regimbau, T., \& Chauvineau, B. 2007, Class. Quantum Grav., 24, 627

Regimbau, T., \& de Freitas Pacheco, J. A. 2006, ApJ, 642, 455 
Regimbau, T., \& Hughes, S. A. 2009, Phys. Rev. D, 79, 062002

Sadowski, A., et al. 2008, ApJ, 676, 1162

Santamaría, L., et al. 2010, Phys. Rev. D, 82, 064016

Wilkins, S. M., Trentham, N., \& Hopkins, A. 2008, MNRAS, 385, 687 


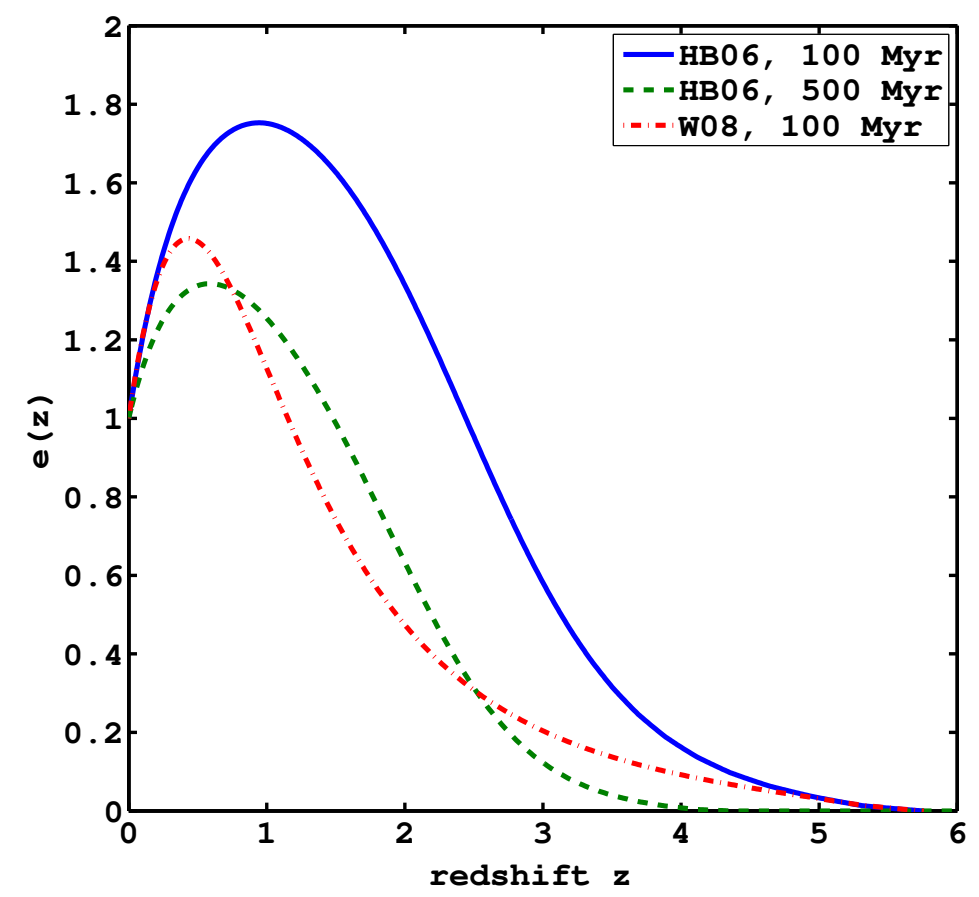

Fig. 1.- Cosmic rate evolution models of BBH coalescences. We show the effects of different SFRs by considering HB06 in Hopkins \& Beacom (2006) and W08 in Wilkins et al. (2008) for a minimal delay time $t_{0}=100 \mathrm{Myr}$. To illustrate the effect of $t_{0}$ we also show the case $t_{0}=500 \mathrm{Myr}$ for the former SFR. 
Table 2: Minimum values of the coalescence rate $r_{0}\left(\mathrm{in} \mathrm{Mpc}^{-3} \mathrm{Myr}^{-1}\right.$ ) to detect the SGWB from coalescing BBHs assuming an average chirp mass $M_{c}=8.7 M_{\odot}$. We explore the result from cross correlation measurements between different pairs of the worldwide network of second-generation ground-based detectors (H,L,V,C,A) and for two approaches of combining 4 detectors (FC and $\mathrm{CP}$ ), taking a SNR of 3 indicate detection.

\begin{tabular}{lccccc}
\hline \hline Pair & A-C & A-H & A-L & A-V & C-H \\
& 1.88 & 0.90 & 0.85 & 2.31 & 3.11 \\
\hline Pair & C-L & C-V & H-L & H-V & L-V \\
& 10.86 & 1.04 & 0.55 & 1.83 & 1.52 \\
\hline Combination & ACHL & ACHV & ACLV & AHLV & CHLV \\
FC & 1.25 & 1.16 & 1.10 & 1.04 & 0.75 \\
CP & 0.40 & 0.57 & 0.56 & 0.38 & 0.45 \\
\hline \hline
\end{tabular}

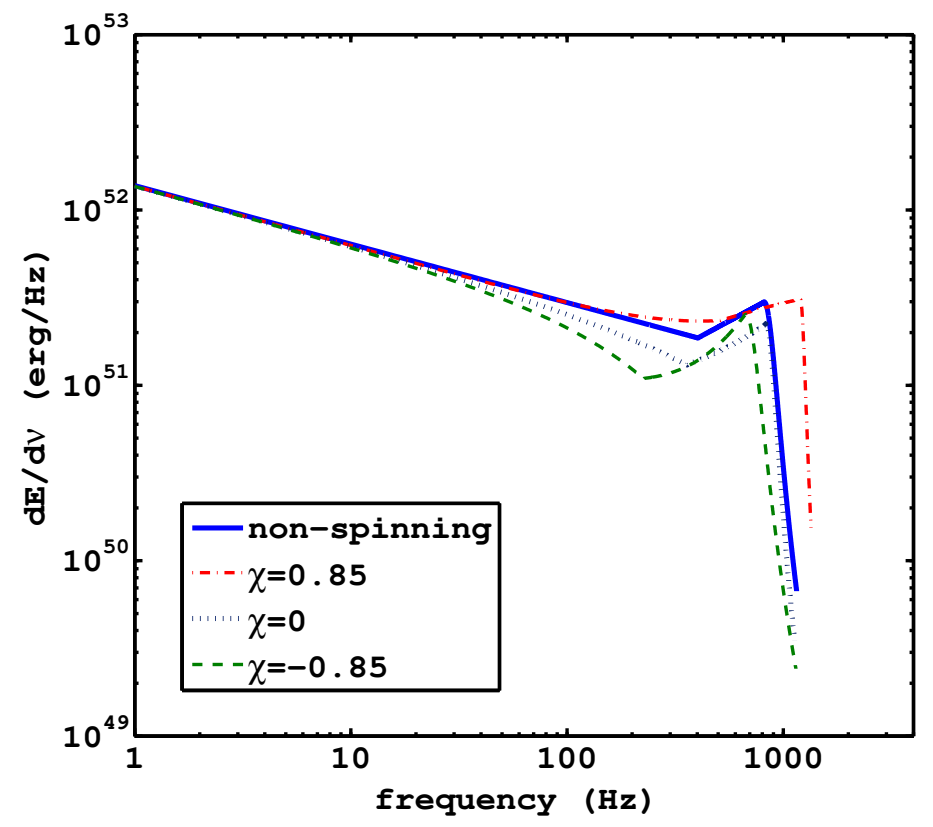

Fig. 2.- GW energy spectra for a $10 M_{\odot}-10 M_{\odot}$ coalescing $\mathrm{BBH}$ in the non-spinning case and cases for non-precessing spins with three values of the single spin parameter $\chi$ (see text). 


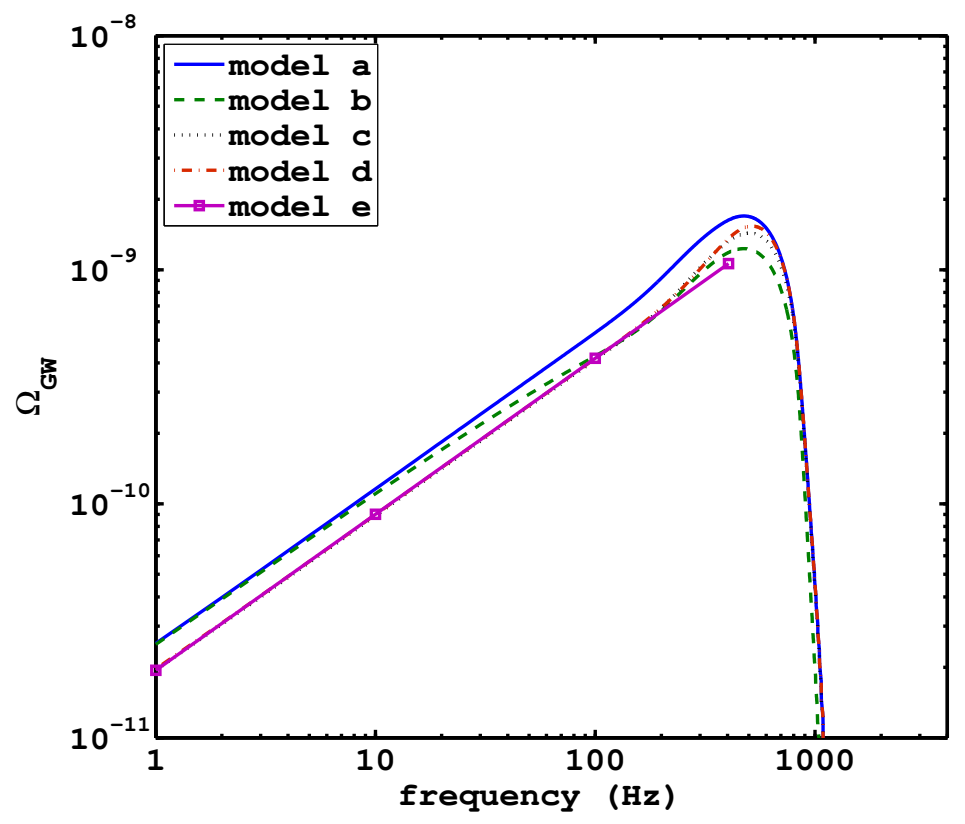

Fig. 3. - The energy density parameter $\Omega_{\mathrm{GW}}$ as a function of observed frequency for the $\mathrm{BBH}$ background corresponding to the five models given in Table 1.

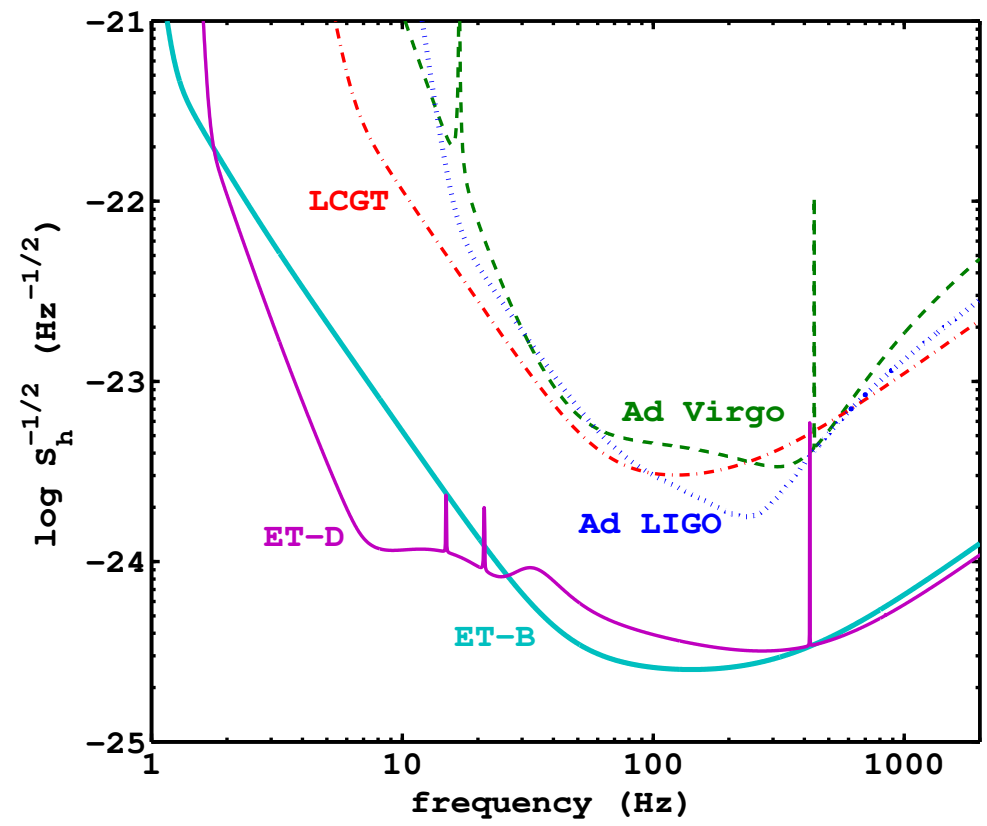

Fig. 4.- The design sensitivity curves for future ground-based detectors: Advanced Virgo (Ad Virgo), LCGT, Advanced LIGO (Ad LIGO) and two possible configurations of the Einstein Telescope, ET-B and ET-D. 


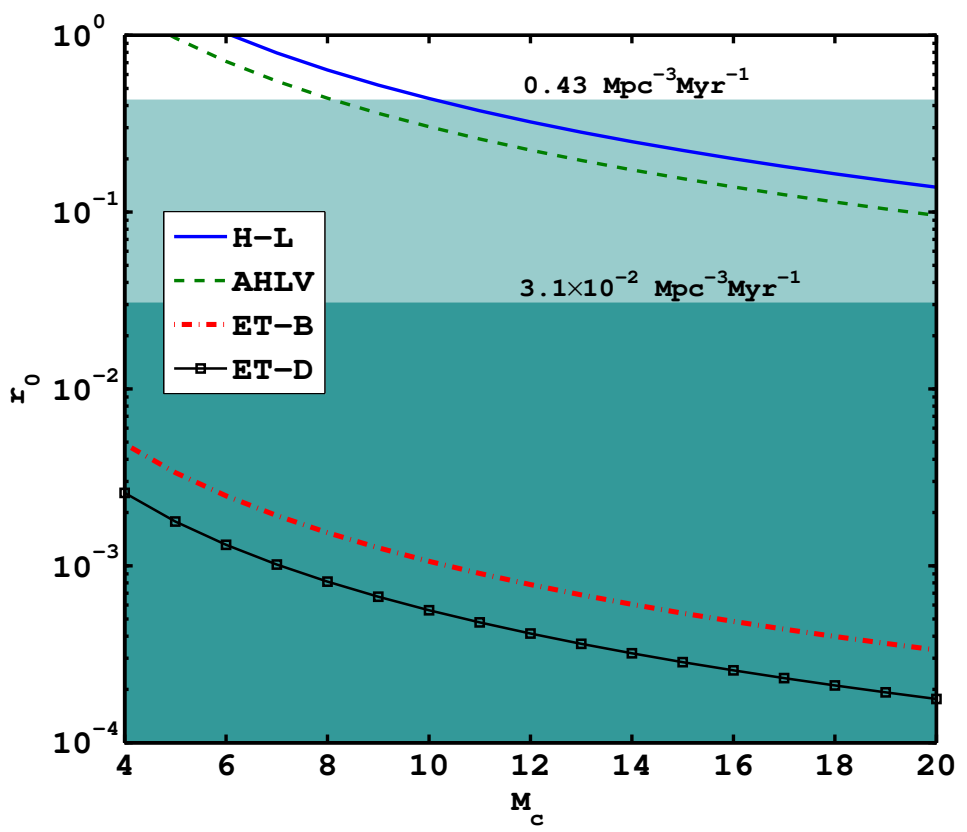

Fig. 5.- The detectable space of parameters for a stochastic background formed by coalescing BBHs: the average chirp mass $M_{c}$ (in $M_{\odot}$ ) and the local rate density $r_{0}\left(\right.$ in $\mathrm{Mpc}^{-3} \mathrm{Myr}^{-1}$ ). The $r_{0}-M_{c}$ curves correspond with those required to produce a $\mathrm{SNR}=3$, through cross correlation measurements by two Advanced LIGO detectors (H-L) and two third generation detectors adopting ET-B/ET-D sensitivities, and the optimal combination of 4 advanced detectors (AHLV). The region above these curves can be considered the detectable parameter space. The two shaded regions correspond to zones encompassed by the two rates $r_{2}$ and $r_{1}$, based on the estimates of Belczynski et al. (2010b). 